\title{
Compactification on non-symmetric six dimensional coset spaces with torsion *
}

\author{
B.P. Dolan ${ }^{1}$, D.C. Dunbar ${ }^{2}$, A.B. Henriques ${ }^{3}$ \\ Department of Physics and Astronomy, University of Glasgow, Glasgow G12 8QQ, UK
}

Received 16 November 1987

\begin{abstract}
The equations of motion of a ten dimensional model based on the Chapline-Manton lagrangian, modified by higher derivative terms, are solved using six dimensional coset spaces with torsion. Minkowski space, anti-de Sitter, de Sitter and Einstein static cosmology with negative curvature are possible four dimensional cosmologies. In all case symmetry breaking schemes $E_{8} \times E_{8} \rightarrow E_{8} \times E_{6}$ with chiral 27's of $E_{6}$ can be obtained.
\end{abstract}

\section{Introduction}

Supersymmetric string theories in ten dimensions [1] are widely regarded as being very promising candidates for a unified theory of gravity and the $S U(3) \times S U(2) \times U(1) \quad$ strong-electro-weak theory. The question of how to extract realistic four dimensional physics from a ten dimensional theory with $S O(32)$ or $E_{8} \times E_{8}$ Yang-Mills symmetry has occupied many authors [2-7] and the path still remains unclear. The device of equating the gravitational connection with the Yang-Mills connection was used by Candelas et al. [2], to compactify six of the ten dimensions on a Calabi-Yau manifold with $S U$ (3) holonomy, to obtain an $E_{6}$ Yang-Mills group in four dimensional Minkowski space. This leads to some very tantalising results, but gives little insight into why four dimensions should be singled out in preference to any other number. Compactifications on six dimensional coset spaces have been examined in [3-7], Castellani and

\footnotetext{
* Work supported in part by NATO grant No RG. 85/0128

1 Permanent Address: Department of Physics, St Patrick's College, Maynooth, Ireland

2 Address after 1/1/88: Department of Mathematical Physics, Royal Holloway and Bedford New College, London, UK

${ }^{3}$ Permanent Address: C.F.M.C., Av Prof Gama Pinto 2, 1699-Lisboa Codex, Portugal
}

Lust [7] performing an analysis of the $\sigma$-model conformal anomaly to show that the anomaly can vanish for the non-symmetric coset spaces $\frac{G_{2}}{S U(3)}$ and $\frac{S U(3)}{U(1) \times U(1)}$, with torsion.

In this paper, an attempt is made to explain the preferential existence of four dimensions dynamically, by giving the three form field $H$, of the gravitational bosonic multiplet, a non zero background value. Equating part of the three form field to the volume form of three dimensional space (in a manner similar to that used in [8] in the context of eleven dimensional supergravity) allows solutions to be constructed with a four dimensional Einstein static cosmology, i.e. $\mathbb{R} \times M_{3}$ where $\mathbb{R}$ is the time-like direction and $M_{3}$ is a maximally symmetric three dimensional manifold. The solutions obtained give negative curvature for $M_{3}$ which is thus a three dimensional hyperboloid, $(\mathrm{Hyp})^{3}$. Maximally symmetric four dimensional space times (Minkowski, anti-de Sitter and de Sitter) will also be considered. In this case the three form field $H$ will only be given a non-zero component in the internal space. The three non-symmetric coset spaces $\frac{G_{2}}{S U(3)}, \frac{S U(3)}{U(1) \times U(1)}$ and $\frac{S p(4)}{S U(2) \times U(1)}$, are examined as candidate internal manifolds. We shall be considering the possibility of allowing non-zero torsion on our manifolds as torsion can be explicitly defined on these non-symmetric coset spaces.

In the original Chapline-Manton theory the role of torsion is clear - we should adopt second order formalism and set the torsion to zero (when the fermion fields are zero); however at the low energy limit of superstrings it is possible that we may find torsion arising from the higher order effects.

When approaching compactification via the $\sigma$ model approach $[6,7]$ some authors have cast the 
$H$ field in the role of the torsion tensor $T_{b c}^{a}$. We also set $T=H$ but having verified that a Minkowski spacetime solution was not allowed decided to investigate the consequences of having a variable torsion. In actual fact, for the ansatz we consider, we still have $H_{a b c}$ proportional to $T_{a b c}$ but the proportionality is not fixed. What is the possible source of this extra torsion? It could come from higher order terms arising from the string theory although we can show, at least to first order, by observing that no 9 of $S O(9)$ exists amongst the first massive level of states, that torsion cannot be a propagating field. At the Chapline-Manton lagrangian level we can also have torsion, via specific non-zero fermion bilinears which have the same tensorial properties as torsion. Even when the background fields are zero, quantum fluctuations of the fermionic fields can produce a torsion as was discussed in [23] where the authors also considered a variable torsion. So we will take the viewpoint that, even if not a free field, there is the possibility that the torsion may be non-zero and we shall attempt to solve the equations of motion with this crucial difference.

The Lagrangian used to obtain the dynamics is that of Chapline and Manton [9] for ten dimensional supergravity coupled to supersymmetric Yang-Mills, modified by the addition of terms arising from superstring theories as suggested in [10] and [12].

The outcome of the analysis is that there are two disconnected one parameter families of solutions for $\mathbb{R} x(\mathrm{Hyp})^{3}$, one joining smoothly onto ten-dimensional Minkowski space, the other joining smoothly onto four-dimensional Minkowski direct producted with one of the three coset spaces, the two limiting cases also being solutions. Anti-de Sitter and de Sitter spaces are also possible solutions. Some possible residual fermion spectra in four dimensions, based on $E_{6}$ as a unification group, are discussed.

Other authors have considered the role of modified Chapline-Manton Lagrangians in compactification, but the present work differs from these in several respects. Randjbar-Daemi et al. [24], modify the Lagrangian by adding a SUSY breaking scalar potential to compactify to four dimensional Minkowski spacetime, but do not consider $R^{2}$ terms. Cosmological implications of gaugino condensates and scalar fields have been considered for compactifications on Calabi-Yau spaces and spheres by Maeda and Pang [25] but neither $R^{2}$ terms nor non-symmetric coset spaces are considered. Maeda and Pollock [26] do consider $R^{2}$ terms, but only for compactifications on CalabiYau spaces, not on other manifolds. Chapline [27] considers canonical connections on non-symmetric coset spaces, but uses only $d H=\frac{\operatorname{Tr}}{30} F \wedge F$, and does not include the tr $R \wedge R$ modification, furthermore the question of dynamics is not addressed at all, only geometry.

General aspects of torsion in superstring theories have been considered by a number of authors. Bars [28] approaches the problem by demanding ten dimensional supersymmetry, and does not consider any dynamics. References [29] and [30] use the two dimensional sigma model approach. Hull [29] considers conditions necessary for finiteness, conformal invariance, anomaly cancellation and world sheet supersymmetry in the sigma model while Rohm and Witten [20] use $N=1$ world sheet supersymmetry as a guide to non zero $H$ configurations. Neither tackles the full ten dimensional equations of motion.

The question of supersymmetry on non-symmetric coset spaces in particular is discussed in [23] and [31].

The layout of the paper is as follows. Section 2 sets up some notation and explains the motivation for our choice of Lagrangian. Section 3 contains a review of non-symmetric coset spaces and their geometrical structures. Section 4 exhibits our ansatz and Sect. 5 details of the solutions. Section 6 contains an analysis of the fermion spectra in four dimensions when our solutions are used to compactify the $E_{8} \times E_{8}$ theory. Section 7 contains a discussion of these solutions. Details of the structure constants and geometrical structures on the three non-symmetric coset spaces under consideration are relegated to an appendix.

\section{Dynamics}

The Lagrangian for ten-dimensional supergravity coupled to supersymmetric Yang-Mills [9] is a ten form in differential form language

$$
\begin{aligned}
\mathscr{L}= & \frac{1}{2} \not \mathscr{R}^{*} 1-\frac{1}{4} e^{2 \sigma} H \wedge^{*} H+\frac{1}{2.30} e^{\sigma} \operatorname{Tr}\left(F \wedge{ }^{*} F\right) \\
& -d \sigma \wedge^{*} d \sigma+\text { Fermionic terms. }
\end{aligned}
$$

We shall assume that the vacuum expectation values of the fermion fields are set to zero and only the bosonic fields have non-zero background values. The field $\sigma$ is the dilaton (scalar) field and $F=d A+A \wedge A$ is the Yang-Mills two form, taking values in the Lie algebra of the gauge group $G,\left(E_{8} \times E_{8}\right.$ or $\left.\frac{S O(32)}{Z_{2}}\right)$. The normalisation is such that $\operatorname{Tr}\left(Q_{i} Q_{j}\right)=-30 N^{2} \delta_{i j}$ with $Q_{i}=-Q_{i}^{+}$in the adjoint representation. $H$ is a three form field, the curl of a two form $B$ modified by the Yang-Mills ChernSimons three form $\omega_{3}^{Y-M}=\frac{1}{30} \operatorname{Tr}\left(A \wedge F-\frac{1}{3} A \wedge A \wedge A\right)$ [9];

$H=d B+\omega_{3}^{Y-M}$. 
This definition of $H$ results in the Bianchi type of identity

$d H=\frac{1}{30} \operatorname{Tr}(F \wedge F)$.

Finally, $\mathscr{R}$ is the Einstein scalar and $*$ is the Hodge duality operator, sending $p$-forms to $(10-p)$ forms (the signature is $(-,+, \ldots,+)$ ). For example, if $e^{A}$, $A=0,1, \ldots, 9$ are orthonormal one-forms, then

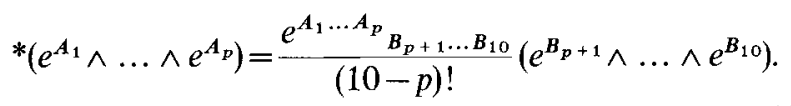

The orthonormal one forms are related to the connection one-forms $\omega_{A B}$ and the torsion two forms $T^{A}$ by

$d e^{A}+\omega^{A}{ }_{B} \wedge e^{B}=T^{A}$,

where $d$ is the exterior derivative.

The curvature two forms are

$R_{A B}=d \omega_{A B}+\omega_{A}^{c} \wedge \omega_{C^{B}}$

and the Einstein scalar is given by

$\mathscr{R}^{*} 1=R_{A B} \wedge *\left(e^{A} \wedge e^{B}\right)$.

Unfortunately it is difficult to find non-trivial solutions to the equations of motion, obtained from $\mathscr{L}$ by varying the fields in the bosonic sector. In fact it has been shown [11] that if it is assumed that tendimensional space-time is split into four dimensional de Sitter, anti-de Sitter or Minkowski space and six compact microscopic dimensions, with no boundary, then there is no solution of the bosonic equations of motion for $\mathscr{L}$. This can be easily seen from the scalar equation. Varying $\sigma$ in $\mathscr{L}$ and integrating by parts gives:

$+\frac{1}{2} e^{2 \sigma} H \wedge{ }^{*} H-\frac{1}{2} e^{\sigma} \frac{\operatorname{Tr}}{30} F \wedge * F-2 d^{*} d \sigma=0$.

Maximal symmetry in four dimensions implies that neither $H$ nor $F$ has a timelike component, hence the $H^{2}$ and $F^{2}$ terms above are positive definite. Maximal symmetry in four dimensions also implies that $\sigma$ must be constant in four dimensional spacetime and can only be a function of the internal coordinates. But the eigenvalues of the Laplacian operator, $d^{*} d$, on a compact manifold with positive definite signature are negative. Hence all three terms in the scalar equation above are of the same sign, and the only solution is if all three vanish.

However, if the Lagrangian above is being considered as the low energy limit of a superstring theory, then there would be other terms, higher order in $\alpha^{\prime} E^{2}$ ( $E$ is the energy scale at which we are working and $\alpha^{\prime}$ is the inverse string tension). The first indication of these came in [12] where cancellation of gravitation anomalies motivated the authors to modify the $\mathrm{H}$-Bianchi identity to include the Lorentz Chern-Simons terms:

$\omega_{3}^{L}=\operatorname{tr}\left(\omega \wedge R-\frac{1}{3} \omega \wedge \omega \wedge \omega\right)$,

leading to

$d H=\frac{1}{30} \operatorname{Tr}(F \wedge F)+\operatorname{tr}(R \wedge R)$

with $\operatorname{tr} R \wedge R \equiv R_{A B} \wedge R^{B A}$ and with anti-hermitian generators of the gauge group. Note that $R^{2}$ contains four derivatives and so is of order $\left(\alpha^{\prime} \mathrm{E}^{2}\right)^{2}$ while $d H$ and $F^{2}$ contain only two and so are of order $\alpha^{\prime} E^{2}$. An $R^{2}$ modification to the dynamics was suggested in [2], in the form of a term like $\frac{1}{2} e^{\sigma} \operatorname{tr}\left(R \wedge^{*} R\right)$ as an extension of the Lagrangian. However this form would be expected to lead to gravitational ghosts and a ghost free form was first propounded by Zweibach [10]:

$\Delta \mathscr{L}=\frac{1}{4} \exp (\sigma) R_{A B} \wedge R_{C D} \wedge^{*}\left(e^{A} \wedge e^{B} \wedge e^{C} \wedge e^{D}\right)$.

In four dimensions, this term would be the Euler density and would locally be a total derivative, thus having no effect on the classical dynamics. However, in ten dimensions it does affect the classical dynamics, as we shall see, avoiding the no go theorem and allowing acceptable compactifications.

This, then, gives us our dynamics. We take the Lagrangian $\mathscr{L}$ and add the $R^{2}$ terms (11) also modifying the $H$-Bianchi identity. The equations of motion for the dilaton, the anti-symmetric tensor field, the Yang-Mills field and the metric are respectively:

$$
\begin{aligned}
& d^{*} d \sigma=\frac{1}{4} \exp (\sigma) H \wedge^{*} H-\frac{1}{4} \exp (\sigma) \frac{\operatorname{Tr}}{30}\left(F \wedge{ }^{*} F\right) \\
& \quad-\frac{1}{8} \exp (\sigma) R_{A B} \wedge R_{C D} \wedge^{*}\left(e^{A} \wedge e^{B} \wedge e^{C} \wedge e^{D}\right) \\
& d\left(e^{2 \sigma *} H\right)=0 \\
& D_{A}\left[\exp (\sigma)^{*} F\right]=-\exp (2 \sigma) F \wedge^{*} H \\
& \frac{1}{2} R_{A B} \wedge^{*}\left(e^{A} \wedge e^{B} \wedge e^{C}\right) \\
& +\frac{1}{4} R_{A B} \wedge R_{D F} \wedge^{*}\left(e^{A} \wedge e^{B} \wedge e^{D} \wedge e^{F} \wedge e^{C}\right)=\tau_{H}^{C}+\tau_{F}^{C}+\tau_{\sigma}^{\mathrm{C}}
\end{aligned}
$$

where the $\tau^{C}$ are the energy-momentum forms defined by

$$
\begin{aligned}
\tau_{H}^{C} & =-\frac{1}{4} \exp (2 \sigma)\left[2\left(i^{C} H\right) \wedge * H-i^{C}\left(H \wedge^{*} H\right)\right] \\
\tau_{F}^{C} & =\frac{1}{2} \exp (\sigma) \frac{\operatorname{Tr}}{30}\left[2\left(i^{C} F\right) \wedge * F-i^{C}(F \wedge * F)\right] \\
\tau_{\sigma}^{C} & =-\left[2\left(i{ }^{C} d \sigma\right) \wedge * d \sigma-i^{C}\left(d \sigma \wedge^{*} d \sigma\right)\right],
\end{aligned}
$$


$i^{c}$ being the interior derivative, i.e. a contraction with the orthonormal tangent space vector $X^{C}$, sending $p$-forms to $(p-1)$ forms. It has the following properties:

$$
\begin{aligned}
& i^{C}\left(e^{A}\right)=\eta^{C A}, \quad i^{C}\left(e^{A} \wedge e^{B}\right)=\eta^{C A} e^{B}-e^{A} \eta^{C B}, \\
& i^{C *}\left(e^{A} \wedge \ldots \wedge e^{B}\right)=*\left(e^{A} \wedge \ldots \wedge e^{B} \wedge e^{C}\right)
\end{aligned}
$$

We shall attempt to solve the above equations of motion, with $H \neq 0$ to provide a splitting of the ten dimensions into $4+6$, the four dimensional spacetime being either maximally symmetric (i.e. de Sitter, anti-de Sitter or Minkowski) or Einstein static. A natural choice for a compact, six dimensional manifold, which admits a non-zero $H$, is one of the three nonsymmetric coset spaces, $\frac{G_{2}}{S U(3)} \approx S^{6}, \frac{S U(3)}{U(1) \times U(1)}$ or $\frac{S p(4)}{S U(2) \times U(1)}$. In the next section we shall review the geometry of coset spaces.

\section{Geometry of coset spaces}

We follow here the work of Salam and Strathdee in [13]. Given two compact Lie groups $W$ and $V$ with $V \subset W$, the set of all left cosets of $W$ with respect to $V$ can be given the structure of a smooth differentiable manifold, with $\operatorname{dimension}=\operatorname{dim} W-\operatorname{dim} V$, which will be denoted by $\frac{W}{V}$. Let $\bar{a}=1, \ldots, \operatorname{dim} V$, $\hat{a}=1, \ldots, \operatorname{dim} W$ and $a=1, \ldots, \operatorname{dim} W-\operatorname{dim} V$. Then $Q_{\vec{a}}$ will denote the generators of $V$ and $Q_{\hat{a}}$ the generators of $W$. Now we set up a co-ordinate system $y$, at least locally, on $\frac{W}{V}$. Each independent value of $y$ labels a distinct left coset in $W$ with respect to $V$. For each value of $y$ we choose an element $L(y)$ of $W$ from the respective coset. Since $\frac{W}{V}$ is a differentiable manifold $L(y)$ is a differentiable function with respect to the co-ordinate system $y$. Hence we can define the $W$-Lie algebra valued one-forms

$e(y)=-\frac{1}{\alpha} L^{-1}(y) d L(y)$

where $d$ is the exterior derivative on $\frac{W}{V}$ and $\alpha$ a real constant. This can be expanded in terms of the generators of $W$, as

$e(y)=e^{\hat{a}} Q_{\hat{a}}=e^{\bar{a}}(y) Q_{\bar{a}}+e^{a}(y) Q_{a}$ where $e^{\bar{a}}(y)$ and $e^{a}(y)$ are one-forms on $\frac{W}{V}$. Since $d^{2}$
$=0$,

$$
\begin{aligned}
d e(y) & =\alpha e(y) \wedge e(y) \\
& =\frac{\alpha}{2} e^{\hat{b}} \wedge e^{\hat{c}}\left[Q_{\hat{b}}, Q_{\hat{c}}\right] \\
& =\frac{\alpha}{2} C_{\hat{b} \hat{c}}{ }^{\grave{a}}\left(e^{\hat{b}} \wedge e^{\hat{\imath}}\right) Q_{\hat{a}} \\
& =\left(d e^{\hat{a}}\right) Q_{\hat{a}}
\end{aligned}
$$

where we have used the commutation relations

$\left[Q_{\hat{a}}, Q_{\hat{b}}\right]=C_{\hat{a} \hat{b}}^{\hat{c}} Q_{\hat{c}}$.

Thus

$d e^{a}=\frac{\alpha}{2} C_{\hat{b} \hat{c}}{ }^{a}\left(e^{\hat{b}} \wedge e^{\hat{c}}\right)$

and

$d e^{\bar{a}}=\frac{\alpha}{2} C_{\hat{b} \hat{c}}^{\bar{a}}\left(e^{\hat{b}} \wedge e^{\hat{c}}\right)$.

A metric on $\frac{W}{V}$ can be manufactured using $e^{a}$ as orthonormal one forms $(a=1, \ldots, \operatorname{dim} W-\operatorname{dim} V)$. Considering the metric as a rank two, symmetric, covariant tensor, we define it to be

$g=\sum_{a=1}^{\operatorname{dim}\left(\frac{w}{v}\right)} e^{a} \otimes e^{a}$

(tangent space indices are raised and lowered with $\left.\eta_{a b}=\operatorname{diag}(+1, \ldots,+1)\right)$.

Torsion two forms can also be defined on $\frac{W}{V}$ using the structure constants of $W$

$T^{a}=\frac{\alpha}{2}(1-\beta) C_{b c}{ }^{a} e^{b} \wedge e^{c}$

where $\beta$ is a real constant. When the $C_{b c}^{a}$ are non-zero, the coset space is said to be non-symmetric. When $C_{b c}^{a}$ are all zero, then

$\left[Q_{b}, Q_{c}\right]=C_{b c}{ }^{a} Q_{a}$

and the coset space is said to be symmetric. In the ansatz that we use, it is crucial that $C_{b c}^{a}$ be non-zero and hence we restrict ourselves to non-symmetric coset spaces. The parameter $\beta$ can take any value. The value $\beta=1$ is the zero torsion case which results in the standard Riemannian connection on a homogeneous space. This case is referred to as the canonical connection of the first kind in the mathematical literature [14].

The case $\beta=0$, the canonical connection of the second kind, results in a connection with holonomy group $V$. In general, arbitrary values of $\beta$ give holo- 
nomy group $S O(n)$ with $n=\operatorname{dim} W-\operatorname{dim} V$. For the three coset spaces on which we focus attention, the values $\beta=1 \pm \sqrt{5}$ are special, since these values result in vanishing Ricci tensor (though the Riemann tensor is still non-zero, of course) [6]. In particular, if $|\beta|$ is large enough, the scalar curvature can become negative. Using (22) and (25) in (5) (noting that $C_{\bar{a} \bar{b}}^{c}=0$ since $V$ is a group), gives

$\omega_{a}^{b}==\frac{\alpha}{2} \beta C_{c a}^{b} e^{c}+\alpha C_{\bar{c} a}^{b} e^{\bar{c}}$

leading to the curvature two forms

$R_{a}^{b}=\frac{\alpha^{2}}{2}\left(C_{\bar{c} a}^{b} C_{f g}{ }^{\bar{c}}+\frac{1}{2} \beta C_{c a}{ }^{b} C_{f g}{ }^{c}+\frac{1}{2} \beta^{2} C_{f a}{ }^{c} C_{g c}{ }^{b}\right) e^{f \wedge g}$

from (6).

For all three possible non-symmetric cases this construction results in an Einstein space for any value of $\beta$. The scalar curvature is the same for all three cases:

$\mathscr{R}=\alpha^{2}\left(2+\beta-\frac{1}{2} \beta^{2}\right)$.

We now turn to the Yang-Mills field on $\frac{W}{V}$. A possible choice of non-zero background field on $\frac{W}{V}$ is to use the remaining part of (19). Let us define $A=-\alpha e^{\bar{a}} Q_{\bar{a}}$ leading to

$F=d A+A \wedge A=-\frac{\alpha^{2}}{2} C_{b c}^{\bar{a}} Q_{\bar{a}}\left(e^{b} \wedge e^{c}\right)$

which takes non-zero values in the Lie algebra of $V$ (indeed the case $\beta=0$, leads to $\omega=A$, the identification of the spin connection with the Yang-Mills connection as suggested in [2]. However for $\beta \neq 0$, $\omega \neq A$ ). There are some subtleties for the case of $\frac{S p(4)}{S U(2) \times U(1)}$, related to the relative normalisations of the $S U(2)$ and $U(1)$ generators, and these are discussed in the appendix. This background Yang-Mills field satisfies the Yang-Mills equations in the background metric (24)

$D^{\hat{*}} F=d^{\hat{*}} F+[A, \hat{*} F]=0$

where ${ }^{*}$ is the six dimensional Hodge duality operator on $\frac{W}{V}$. (The Bianchi identity $D F=0$ is trivial). Using the structure constants for the three non-symmetric coset spaces as given in the appendices, we find that the energy momentum tensor $\left(\tau_{F}\right)^{a}{ }_{b}$, given by $\tau_{F}^{a}=$ $\left(\tau_{F}\right)_{b}^{a \hat{*}} e^{b}$, for this Yang-Mills field is diagonal.

\section{Ansatz}

We are now ready to write down an ansatz for a solution of the equations of motion for $\mathscr{L}+\Delta \mathscr{L}$, with the topology of $M_{4} \times\left(\frac{W}{V}\right)$ and $M_{4}$ one of the two
possible space times

i) maximally symmetric, i.e. Minkowski, de Sitter or anti-de Sitter,

ii) Einstein static cosmology, with a maximally symmetric three space, $\mathbb{R} \times M_{3}$ (where $\mathbb{R}$ is the timelike direction). Let $0^{\prime}, 1^{\prime}, 2^{\prime}, 3^{\prime}$ or $\mu, v, \ldots$ denote four dimensional space-time indices, with $e^{\mu}$ the orthonormal one forms for the four dimensional metric, and $1, \ldots, 6$ or $a, b, \ldots$ denote the internal indices. The orientation is given by ${ }^{*} 1=e^{1} \wedge e^{2} \ldots e^{6} \wedge e^{1^{\prime}} \wedge e^{2^{\prime}} \wedge e^{3^{\prime}} \wedge e^{0^{\prime}}$. The scalar field $\sigma$ will be everywhere a constant. It can be scaled out of all the fields (absorbed into the other constants of the ansatz) but it should be remembered that ultimately it is a degree of freedom that can be used to set the scale. As for the three form $H$, in case i) it must vanish on four dimensional spacetime in accordance with the maximal symmetry of space-time, but in case ii) it could have a component proportional to the volume element of $M_{3}$. Its internal components will be taken to be $h C_{a b c}$ which for a symmetric coset space vanishes of course. This ansatz can be written as

$H=h_{0} e^{1^{\prime}} \wedge e^{2^{\prime}} \wedge e^{3^{\prime}}+\frac{h}{3 !} C_{a b c} e^{a} \wedge e^{b} \wedge e^{c}$

where $h$ and $h_{0}$ are real constants ( $h_{0}$ must vanish for case i)). Inserting the structure constants of the three non-symmetric coset spaces of the appendix, the energy momentum tensor $\tau_{b}^{a}\left(\tau^{a}=\tau^{a *}{ }_{b} e^{b}\right)$ reduces to a block product of constants times the unit matrix.

The ansatz for the Yang-Mills field is as given in the last section (30). It is assumed to be zero on four dimensional space-time. For completeness, we will also consider the case where the background Yang-Mills field vanishes identically, on $\frac{W}{V}$.

Finally we consider the metric. This will take the direct product form with $e^{\mu}$ independent of the internal space and $e^{a}$ independent of the external space. Four dimensional space-time will be assumed torsion free, with a cosmological constant allowed in case i). Thus the curvature two forms are

$R^{\mu \nu}=\Lambda e^{\mu} \wedge e^{v} \quad \mu, v=0^{\prime}, 1^{\prime}, 2^{\prime}, 3^{\prime}$ 
with $A=0,>0,<0$ for Minkowski, de Sitter and anti-de Sitter spaces respectively.

For case ii), Einstein static universe, we have

$R^{0^{\prime} i^{\prime}}=0$

$R^{i^{\prime} j^{\prime}}=\lambda e^{i^{\prime}} \wedge e^{j^{\prime}}$

with $i^{\prime}, j^{\prime}=1^{\prime}, 2^{\prime}, 3^{\prime} . \lambda=0,>0,<0$ for Minkowski, $\mathbb{R} \times S^{3}$ and $\mathbb{R} \times(\mathrm{Hyp})^{3}$ spaces respectively.

The internal metric and connection are given by (23) and (27), leading to curvature two forms (28). The free parameters in the ansatz are thus $h_{0}, h, \alpha$, $\beta$ and $A($ or $\lambda)$ and our task will be to solve (10), (12), (13), (14) and (15) with this ansatz.

\section{Solutions}

The equations shall be treated in order of complexity. First the equation of motion for the antisymmetric tensor

$d^{*} H=0$

It is not difficult to show that, with the choice (32), this equation is automatically satisfied, and does not impose any constraints on the ansatz.

The Yang-Mills equation is given by

$D^{*} F=-F \wedge{ }^{*} H$.

The left-hand side vanishes, as discussed in Sect. 3, and it is not difficult to show that the right hand side is identically zero for our ansatz. Hence this equation is also automatic and does not impose any constraints.

The Bianchi identity

$d H=\frac{1}{30} \operatorname{Tr}(F \wedge F)+\operatorname{tr}(R \wedge R)$

does, however, provide a constraint. The contribution of $R_{\mu \nu}$ vanishes for both cases (i) and (ii) as does the $M_{4}$ component of $d H$, leaving only internal components for (10). A four form equation on a six dimensional manifold has $\left(\begin{array}{l}6 \\ 4\end{array}\right)=15$ components but remarkably, with the above ansatz, the left and right hand sides have exactly the same form, leaving only one constraint. (For the case of $\frac{S p(4)}{S U(2) \times U(1)}$ this requires a particular choice of relative normalisation of the generators of $S U(2)$ and $U(1)$, as explained in the appendix). Equation (10) reduces to a single constraint which we write as

$h=\alpha^{3}\left(N^{2}-L+\frac{3}{4} \beta^{2} K\right) \equiv \alpha^{3} R(\beta)$.
We have used the Jacobi identity for the structure constants and the fact that they obey $K \delta^{a b}=C_{c d}^{a} C^{c d b}$ and $L \delta^{a \bar{b}}=C_{c d}^{a} C^{c d \bar{b}}$ with $K$ and $L$ as given in the appendix. Note that, with $F^{\bar{a}}$ as in (30), it is shown in the appendix that $N^{2}=L$, so $h=\frac{3 K}{4} \alpha^{3} \beta^{2}$ thus the canonical connection of the 2 nd kind $(\beta=0)$ is the one which leads to $d H=\frac{1}{30} \operatorname{Tr}(F \wedge F)-\operatorname{tr}(R \wedge R)=0$, and results in anomaly cancelation in four dimensions [15]. This is as one would expect, since $\beta=0$ is the choice of gravitational connection which results in $F=R$. Other choices of $\beta$ upset this equality.

Before considering the scalar and Einstein equations it will be useful to introduce a few definitions concerning the curvature two-forms in the internal space:

$$
\begin{aligned}
& R_{a b} \wedge *\left(e^{a} \wedge e^{b}\right)=\frac{\alpha^{2}}{2} Q(\beta)^{*} 1, \quad a, b=1 \ldots 6 \\
& R_{a b} \wedge R_{c d} \wedge^{*}\left(e^{a} \wedge e^{b} \wedge e^{c} \wedge e^{d}\right)+2 \frac{\operatorname{Tr}}{30}\left(F \wedge^{*} F\right) \\
& =\alpha^{4} P(\beta)^{*} 1, \quad a, b, c, d=1 \ldots 6
\end{aligned}
$$

Where, upon explicit evaluation using the structure constants, we find that $Q(\beta)=\left(4+2 \beta-\beta^{2}\right)$ and $P(\beta)$ $=\left(\frac{1}{6}\right)\left(\beta^{4}-2 \beta^{3}-3 \beta^{2}+8 \beta+A\right), A$ being given by

$\frac{A}{6}=\left[v L^{2}\left(1+\frac{v}{3}\right)-N^{2} v L\right]$

with $L$ and $N^{2}$ as defined previously and $v=\operatorname{dim} V$. When $F^{\bar{a}}=0 A=11$ for $\frac{G_{2}}{S U(3)}, A=20$ for $\frac{S U(3)}{U(1) \times U(1)}$ and it is not defined for $\frac{S p(4)}{S U(2) \times U(1)}$. When $F^{\bar{a}}$ is non-zero, as in (30), $A=8$ for all three cosets (see appendix).

Now consider the scalar equation which we will write in the form

$$
\begin{aligned}
& \frac{1}{2} R_{A B} \wedge R_{C D} \wedge *\left(e^{A} \wedge e^{B} \wedge e^{C} \wedge e^{D}\right) \\
& =H \wedge * H-\frac{1}{30} \operatorname{Tr}(F \wedge * F) .
\end{aligned}
$$

Inserting the ansatz corresponding to case i) of a maximally symmetric space-time, with $h_{0}=0$, this equation can be written as

$$
\frac{1}{2} \alpha^{4} P(\beta)+6 A \alpha^{2} Q(\beta)=K h^{2}+12 \Lambda^{2},
$$

For the Einstein static case ii), the result is

$\frac{1}{2} \alpha^{4} P(\beta)+3 \lambda \alpha^{2} Q(\beta)=h_{0}^{2}+K h^{2}$.

It remains to consider the Einstein equations, (15). Inserting our ansatz for $R_{\mu \nu}, R_{a b}$, and $F$ reduces these equations to polynomial constraints on the ansatz parameters, $h_{0}, \alpha, \beta$ and $A$ (or $\lambda$ ). For case (i) we 
obtain two single constraints, arising from the internal and external equations, and for case (ii) three single constraints arising from the internal, the 3 -space and time equations. Writing them explicitly, we have for case i):

$3 A+\frac{\alpha^{2}}{4} Q(\beta)=\frac{h^{2} K}{4}-\frac{3}{2} \alpha^{2} \Lambda Q(\beta)-\frac{1}{4} \alpha^{4} P(\beta)$,

$6 \Lambda+\frac{\alpha^{2}}{6} Q(\beta)=-6 A^{2}-2 \Lambda \alpha^{2} Q(\beta)-\frac{\alpha^{4}}{12} P(\beta)$,

For case ii), (15) will give three polynomial equations:

$3 \lambda+\frac{\alpha^{2}}{4} Q(\beta)=\frac{1}{4}\left(h^{2} K+h_{0}^{2}\right)-\frac{3 \lambda}{2} \alpha^{2} Q(\beta)-\frac{1}{4} \alpha^{4} P(\beta)$,

$\lambda+\frac{\alpha^{2}}{4} Q(\beta)=\frac{1}{4}\left(h^{2} K-h_{0}^{2}\right)-\frac{\alpha^{2}}{2} \lambda Q(\beta)-\frac{1}{4} \alpha^{4} P(\beta)$,

$3 \lambda+\frac{\alpha^{2}}{6} Q(\beta)=\frac{1}{4} h_{0}^{2}-\alpha^{2} \lambda Q(\beta)-\frac{\alpha^{4}}{12} P(\beta)$.

The case of a zero background Yang-Mills field can be extracted from the above equations simply by setting $N^{2}=0$. Eliminating $h$ from the equations of motion, using (35) we are left with the following parameters and constraints:

case (i) $h, \alpha, \beta$ and $\Lambda$ must satisfy (40), (42), (43) and (35).

case (ii) $h, \alpha, \beta, \lambda$ and $h_{0}$ must satisfy (41), (44), (45), (46) and (35).

These equations are not linear so there is no guarantee of success. We start by considering case i) first. Equations (40), (42) and (43) can be combined to give

$\frac{h^{2}}{3}=-\left(\alpha^{2} Q(\beta)+24 \Lambda\right)$

which substituted back into (42) gives

$9 A+\frac{\alpha^{2}}{2} Q(\beta)=-\frac{3}{2} \alpha^{2} \wedge Q(\beta)-\frac{1}{4} \alpha^{4} P(\beta)$.

We can still combine (42) and (43) to obtain

$\Lambda\left[4 \Lambda+2+\alpha^{2} Q(\beta)\right]=0$,

giving $\quad \Lambda=0$ (Minkowski space)

or

$\Lambda=-\left\{\frac{1}{2}+\frac{\alpha^{2} Q(\beta)}{4}\right\}$.

Treating Minkowski space first, inserting $A=0$ back into (48) gives

$\alpha^{2}=-2 Q(\beta)\{P(\beta)\}^{-1}$.

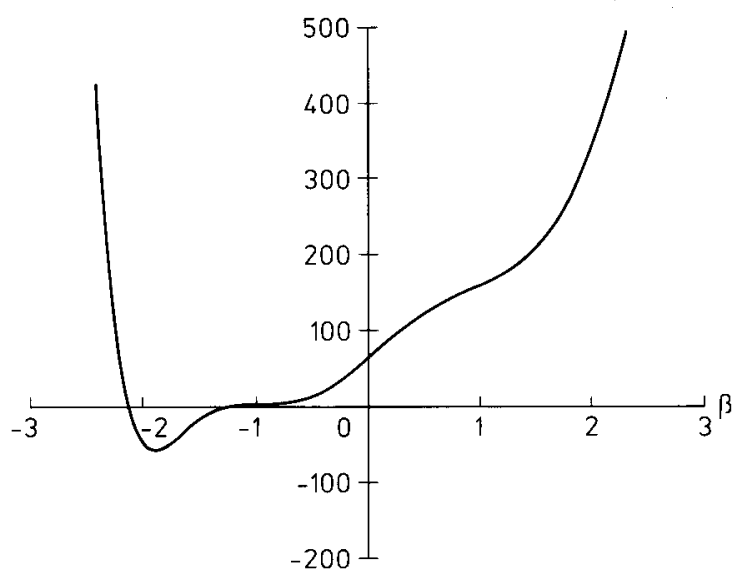

Fig. 1. Graph of the function which determines whether any Minkowski solutions exist in Sect. 5 . We give here the function given in (52) for the case when the Yang-Mills fields are non-zero as in (30). To have a solution of the equations of motion this function must be zero. As we can see there are two roots, at $\beta=-2.13$ and at $\beta=-1.3$. At $\beta=-1.3$ we do not have $\alpha^{2} \geqq 0$ and $h^{2}>0$ so we do not have a valid solution. At $\beta=-2.13$ we find $\alpha^{2}=3.3$ and $h^{2}=48$ so we do have a valid solution at $\beta=-2.13$

Thus everything is determined in terms of $\beta$, which must be chosen to satisfy (35)

$4 K Q(\beta) R^{2}(\beta)+\{P(\beta)\}^{2}=0$.

Inserting the group theory factors into (51), we find that there are no real solutions for $N^{2}=0\left(F^{a}=0\right)$, for any of the three non-symmetric coset spaces, but with $F^{a} \neq 0$ all coset spaces give the same polynomial in (51).

$3 \beta^{4}\left(\beta^{2}-2 \beta-4\right)-\left(\beta^{4}-2 \beta^{3}-3 \beta^{2}+8 \beta+8\right)^{2}=0$

(A graph of this function is given in Fig. 1.) The real solutions are $\beta=2.13,-1.24$ but (47) and (50) must be checked to make sure that $\alpha^{2}>0$ and $h^{2}>0$. We find only one acceptable value for $\beta$ :

$\beta=-2.13 \quad \alpha^{2}=3.3 \quad h^{2}=48 \cdot 0$

Now consider the situation when $A \neq 0$. Inserting $\Lambda$, from (49), back into (48) gives

$\alpha^{2}=\frac{-5 Q(\beta) \pm\left\{2 Q^{2}(\beta)+18 P(\beta)\right\}^{\frac{1}{2}}}{\left\{\frac{3}{2} Q^{2}(\beta)-P(\beta)\right\}}$.

Again, everything is determined in terms of $\beta$ which has to satisfy (35)

$\frac{\alpha^{3}}{3} R^{2}(\beta)=5 \alpha^{2} Q(\beta)+12$. 
Using the full expressions for $P(\beta), Q(\beta)$ and $R(\beta)$ given in (35) and (38), gives (54) and (55) as

$\alpha^{2}=-\frac{30\left(\beta^{2}-2 \beta-4 L\right) \pm 6\left[3\left(\beta^{2}-2 \beta^{3}-3 \beta^{2}+8 \beta+A\right)-2\left(\beta^{2}-2 \beta-4\right)^{2}\right]^{\frac{1}{2}}}{\left[\beta^{4}-2 \beta^{3}-3 \beta^{2}+8 \beta+A-9\left(\beta^{2}-2 \beta-4\right)^{2}\right]}$

and

$\frac{\alpha^{6}}{16}\left(\beta^{2}+4 N^{2}-4 L\right)^{2}+15 \alpha^{2}\left(\beta^{2}-2 \beta-4\right)-36=0$.

When $F^{a} \neq 0 \quad A=8$ for all three non-symmetric coset spaces. For this situation the function in (57) has zeros at three values of $\beta$ (found numerically). However requiring $\alpha^{2} \geqq 0$ in (56) and $h^{2} \geqq 0$ in (47) eliminates one of the roots, leaving us with two roots

Root $1 \alpha^{2}=1.945, \quad \beta=3.16, \quad h^{2}=15.25, \quad A=-7.95$

Root $2 \alpha^{2}=0.218, \quad \beta=4.99, \quad h^{2}=0.1324$,

$\Lambda=+1.12$.

We see that for Root $1 A<0$, i.e. we have an anti-de Sitter space and for Root $2 A>0$, i.e. we have a de Sitter space.

When $F^{\bar{a}}=0, A$ is given in (38) for $\frac{G_{2}}{S U(3)}$ and $\frac{S U(3)}{U(1) \times U(1)}\left(\frac{S p(4)}{S U(2) \times U(1)}\right.$ is not a valid solution in this case). These values of $A$ solve (57) and (56) with the following (numerically found) values

$$
\begin{array}{llll}
\alpha^{2}=1.1 & \beta=-1.73 & h^{2}=0.019 & A=1.188 \\
\alpha^{2}=5.5 & \beta=-1.12 & h^{2}=26.1 & \Lambda=-14.6 \\
\alpha^{2}=2.89 & \beta=2.89 & h^{2}=4.88 & A=-26.6 \\
\alpha^{2}=0.205 & \beta=5.09 & h^{2}=0.084 & \Lambda=1.15,
\end{array}
$$

for $\frac{S U(3)}{U(1) \times U(1)}$

and

$$
\begin{aligned}
& \alpha^{2}=2.095 \quad \beta=-1.474 \quad h^{2}=0.265 \quad \Lambda=1.042 \\
& \alpha^{2}=2.32 \quad \beta=3.093 \quad h^{2}=19.17 \quad A=-10.31 \\
& \alpha^{2}=0.2134 \quad \beta=5.016 \quad h^{2}=0.1184 A=1.129 \text {, } \\
& \text { for } \frac{G_{2}}{S U(3)} \text {. }
\end{aligned}
$$

We now turn to the Einstein static universe. It is not difficult to show that the scalar equation (41) is a direct consequence of the Einstein equations (44)(46), therefore it is sufficient to solve (44)-(46) only.
These three equations can be re-arranged as

$$
\begin{aligned}
& \frac{1}{4} h_{0}^{2}=3 \lambda+\frac{\alpha^{2} Q(\beta)}{6}+\frac{\alpha^{4}}{12} P(\beta)+\alpha^{2} Q(\beta) \lambda, \\
& h^{2}=\alpha^{2} Q(\beta)+2 \alpha^{4} P(\beta)+6 \alpha^{2} Q(\beta) \lambda, \\
& 6 \lambda=-\left[2 \alpha^{2} Q(\beta)+\alpha^{4} P(\beta)\right]\left[\alpha^{2} Q(\beta)+4\right]^{-1} .
\end{aligned}
$$

These give $h^{2}, h_{0}^{2}$ and $\lambda$ in terms of $\alpha$ and $\beta$. The Bianchi identity (35), gives one constraint on $\alpha$ and $\beta$

$$
\begin{aligned}
\alpha^{2} Q(\beta) & -\frac{1}{4} \alpha^{4} Q^{2}(\beta)+2 \alpha^{4} P(\beta)+\frac{\alpha^{6}}{4} Q(\beta) P(\beta) \\
= & \frac{1}{8} \alpha^{6} R^{2}(\beta)\left[\alpha^{2} Q(\beta)+4\right] .
\end{aligned}
$$

Inserting the expressions for $P(\beta), Q(\beta)$ and $R(\beta)$, we get a cubic polynomial in $\alpha^{2}$ from (64)

$a(\beta) \alpha^{6}+b(\beta) \alpha^{4}+c(\beta) \alpha^{2}+d(\beta)=0$,

where

$$
\begin{aligned}
a(\beta)= & \frac{1}{4}\left(\frac{\beta^{2}}{4}-L+N^{2}\right)^{2}\left(\beta^{2}-2 \beta-4\right) \\
b(\beta)= & -\frac{1}{24}\left(\beta^{2}-2 \beta-4\right)\left(\beta^{4}-2 \beta^{3}-3 \beta^{2}+8 \beta+A\right) \\
& -\left[\frac{\beta^{2}}{4}-L+N^{2}\right]^{2} \\
c(\beta)= & \frac{1}{3}\left(\beta^{4}-2 \beta^{3}-3 \beta^{2}+8 \beta+A\right)-\frac{1}{4}\left(\beta^{2}-2 \beta-4\right)^{2} \\
d(\beta)= & -\left(\beta^{2}-2 \beta-4\right) .
\end{aligned}
$$

For $F^{\bar{a}} \neq 0$ all three coset spaces give solutions in a piecewise continuous range of $\beta,-2.15<\beta<-1.24$ and $3.26<\beta<4.8$, compatible with $\alpha^{2}>0, h^{2}>0$, as shown in Fig. $2 \mathrm{a}$ and b. For $F^{\bar{a}}=0, \frac{G_{2}}{S U(3)}$ and $\frac{S U(3)}{U(1) \times U(1)}$ also give solutions in a continuous range of $\beta$. For $F^{a} \neq 0$, solutions exist (Fig. $2 a$ ) in a smooth set from the case of both internal and external spaces being highly curved to the case of a highly curved internal space but flat Minkowski $4-D$ space-time.

To summarise, we have a large class of solutions to the equations of motion. When $F^{\bar{a}} \neq 0$ four dimensional space-time can be Minkowski, anti-de Sitter, de Sitter or spatially unbounded Einstein static and the internal space can be any of the three non-symmetric coset spaces. When $F^{\vec{a}}=0$, only anti-de Sitter and spatially unbounded Einstein static solutions are 

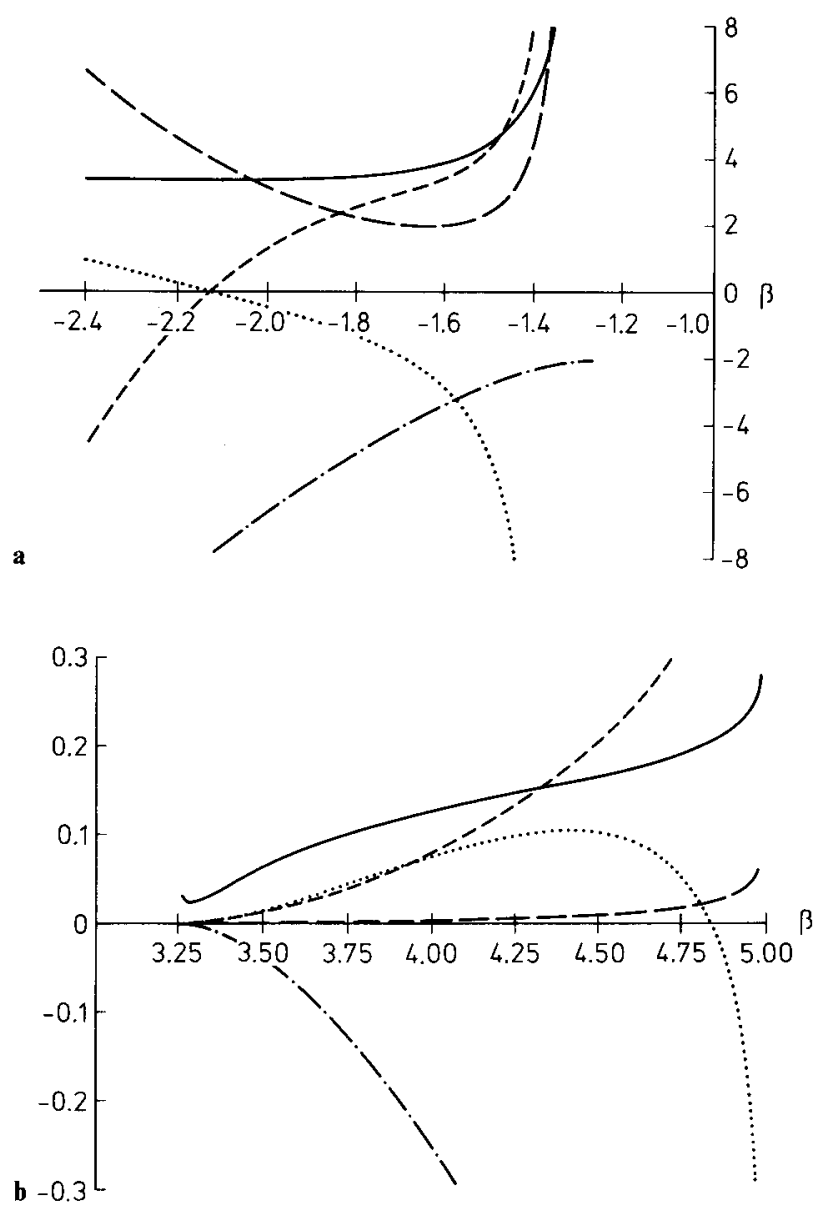

Fig. 2a, b. Details of the solutions for the case where space-time is Einstein static. a First region, $\mathbf{b}$ second region. For the case where space-time is Einstein static we find our solutions are a one parameter family. If we take $\beta$ as the parameter we find we have valid solutions for $-2.15<\beta<-1.24$ and $3.26<\beta<4.8$. We present in $\mathbf{a}$ and $\mathbf{b}$ the variables $h^{2}, h_{0}^{2}, \alpha^{2}, \lambda$ and $\mathbb{R}$ (the internal curvature) as a function of $\beta$. Notice, in a, that there is a value where $h_{0}=\lambda=0$ and we reduce to the Minkowski space-time solutions. For the $-v e$ region $\lambda$ is $-v e$ so space-time is $\mathbb{R} \times(\mathrm{Hyp})^{3}$ and for the + ve region $\lambda$ is +ve so space-time is $\mathbb{R} \times S^{3}$

$-\longrightarrow-3 \times h^{2}, \cdots 9 \times h_{0}^{2},-\alpha^{2},--\longrightarrow \mathbb{R},----\lambda$

allowed with $\frac{G_{2}}{S U(3)}$ or $\frac{S U(3)}{U(1) \times U(1)}$ internal space. Thus a Minkowski four-dimensional space-time requires both a non-zero background value for the Yang-Mills field $F^{\bar{a}}$ and $T \neq H$. It can be verified that $T=H$ only in case (ii) of a $\mathbb{R} \times(\mathrm{Hyp})^{3} \times \frac{W}{V}$ solution; in particular Minkowski four dimensional space-time is not allowed, when $T=H$.

\section{Fermion spectra}

We now discuss what the residual four dimensional fermion spectra would look like under the above com- pactification schemes, when the original Yang-Mills gauge group is $E_{8} \times E_{8}$.

When $F^{\bar{a}}=0$, the gauge group is unbroken, and the four dimensional fermion spectrum is real. Thus $F^{\bar{a}}=0$ does not appear to give any good physics in four dimensions. Interestingly enough, $F^{\bar{a}}=0$ is forbidden dynamically if we wish to have four dimensional Minkowski space.

When $F^{a} \neq 0$, the fermion spectrum depends on the coset space used, and we shall treat each one in turn.

$\frac{G_{2}}{S U(3)}$

Embedding $S U(3)$ into $E_{8}$ breaks it down to $E_{6}$ as $E_{8}$ has $S U(3) \times E_{6}$ as a maximal subgroup [2]. Under this embedding the 248 of $E_{8}$ decomposes as

$$
248 \rightarrow(1,78)+(8,1)+(3,27)+(\overline{3}, \overline{27}) .
$$

The fermions that we wish to identify as the observed chiral families in four dimensions are the 27 and $\overline{27}$ 's of $E_{6}$. The imbalance between massless 27's and $\overline{27}$ 's in four dimensions is given by the imbalance between massless 3 and $\overline{3}$ 's of $S U(3)$ in the background field on the internal manifold. This is given by the index theorem for a six dimensional manifold

$n_{+}-n_{-}=\frac{1}{6} \int\left(3 c_{3}-3 c_{2} c_{1}+c_{1}^{3}\right)-\frac{1}{24} \int p_{1} c_{1}$,

where $c_{i}$ is the $i$ th Chern class and $p_{1}$ is the first Pontrjagin class. Since $\frac{G}{S U(3)} \approx S^{6}[16]$ the first Pontrjagin class $p_{1}=0$. Also the Chern class $c_{1}=0$ for a $S U(3)$ field, hence

$n_{+}-n_{-}=\frac{1}{2} \int_{S^{6}} c_{3}$.

When $\beta=0$ (canonical connection of the second kind) $F=R$ and the first Chern class is identical to the Euler class. Since the Euler characteristic of $S^{6}$ is 2, we get

$n_{+}-n_{-}=1$

For different $\beta$ we must get the same value of $n_{+}-n_{-}$, since this is a topological invariant. That $n_{+}-n_{-}$ $=\frac{1}{2} \times($ Euler characteristic) for a $S U(3)$ bundle was used in [2]. This value differs from that of [5].

One excess chiral fermion in the 3 of $S U(3)$ on the internal space leads to one massless chiral fermion family on four dimensional space-time in the 27 of $E_{6}$. Thus, we have an imbalance of a single 27 of $E_{6}$ in four dimensions. Of course, we would like three or four families, and it may be possible to gain a horizontal symmetry from the $G_{2}$ group of isometries 
associated with $\frac{G_{2}}{S U(3)}$. However, if such a mechanism were to work, one would expect a minimum of seven families (seven being the lowest dimensional representation of $G_{2}$ ) which is incompatible with cosmology (see [17] and references therein). If this idea were to work, we must find some way of breaking the $G_{2}$ down to some smaller group. At present we have no suggestions as to how this may be done.

\section{$\frac{S p(4)}{S U(2) \times U(1)}$}

The fermion spectrum on this manifold has been considered in [5], where the topology is discussed. Considering $S p(4)$ as an $S^{3}$ bundle over $S^{7}$ (it has the same cohomology as $S^{3} \times S^{7}$ ), the coset space is formed by allowing $S U(2)$ to act on $S^{7}$ and $U(1)$ to act on $S^{3}$ so as to induce the fibrations $S^{7} \rightarrow S^{4}$ and $S^{3} \rightarrow S^{2}$, resulting in a $S^{2}$ bundle over $S^{4}$ (since $\pi_{3}\left(S^{2}\right)=\mathbb{Z}[18]$ these bundles are classified by the integers). Hence $\frac{S p(4)}{S U(2) \times U(1)}$ is an $S^{2}$ bundle over $S^{4}$.

Embedding a non-zero background $S U(2) \times U(1)$ field into one $E_{8}$ produces the following decomposition

$$
\begin{aligned}
E_{8} \rightarrow \quad & E_{7} \times S U(2) \\
(248) & (133,1)+(1,3)+(52,2) \\
\rightarrow E_{6} & \times U(1) \times S U(2) \\
& (78,1)_{0}+(27,1)_{-2}+(\overline{27}, 1)_{2}+(1,1)_{0}+(1,3)_{0} \\
& \left.+(27,2)_{1}+(\overline{27}, 2)_{-1}+(1,2)_{3}+1,2\right)_{-3}
\end{aligned}
$$

The $(27,1)_{-2},(27,2)_{1}$, and their complex conjugates would the interpreted as fermion families in four dimensions.

We now examine the index theorem to discover the excess of (27)'s over (27)'s. Since the first Pontrjagin class of $S^{4}$ vanishes, the index theorem for a fermion with $U(1)$ charge $p$ in a background $S U(2) \times U(1)$ bundle over $\frac{S p(4)}{S U(2) \times U(1)}$ is

$$
\begin{aligned}
n_{+}-n_{-} & =\int \operatorname{ch}[S U(2) \times U(1)] \\
& =\int \operatorname{ch}[S U(2)] \wedge \operatorname{ch}[U(1)] \\
& =\int\left(-c_{2}[S U(2)]+\frac{1}{2}\left(c_{1}[S U(2)]\right)^{2}\right) \wedge c_{1}[U(1)] \\
& =-\int c_{2}[S U(2)] \wedge c_{1}[U(1)] \\
& =-m n p
\end{aligned}
$$

(the $U(1)$ charge $p=1$ since the $(27,1)_{-2}$ does not couple to the $S U(2)$ field at all.) where $m=$ monopole charge on $S^{2}$ and $n=$ instanton number on $S^{4}$. This formula disagrees by a factor of $\frac{1}{2}$ with that of [5] e.g. if $m n=2$ there is no massless excess of $(27)_{-2}$ 's over $(\overline{27})_{2}$ 's and 2 more massless $(\overline{27})_{-1}$ 's than $(27)_{1}$ 's in four dimensions.

$\frac{S U(3)}{U(1) \times U(1)}$

The fermion spectrum on this manifold has been considered in [5] and [19], but here we shall consider an alternative scheme.

$$
\frac{S U(3)}{U(1) \times U(1)} \text { can be constructed as a } C P^{1} \text { bundle }
$$

over $C P^{2}$. This structure is obtained by considering $S U(3)$ as a $S^{3}$ bundle over $S^{5}$ (There is one and only one non-trivial $S^{3}$ bundle over $S^{5}$ since $\pi_{4}\left(S^{3}\right)=\mathbb{Z}_{2}$ [18], this is $S U(3))$. By allowing one $U(1)$ to act on $S^{5}$ and the other on $S^{3}$, so as to induce Hopf fibrations $S^{3} \rightarrow S^{2} \approx C P^{1}$ and $S^{5} \rightarrow C P^{2}$, we reduce the $S^{3}$ bundle over $S^{5}(S U(3))$ to a $S^{2}$ bundle over $C P^{2}$ $\left(\frac{S U(3)}{U(1) \times U(1)}\right)$

Embedding a $U(1) \times U(1)$ non-zero background into $E_{8}$ gives two of the gauge bosons in the Cartan subalgebra of $E_{8}$ a mass, see Ref. [15], due to the expectation values of the Chern-Simons terms in the field strength of the antisymmetric tensor. There are various ways of embedding $U(1) \times U(1)$ into $E_{8}$. We shall discuss one which gives $E_{6}$ as a residual gauge group in four dimensions. $E_{8}$ contains $E_{7} \times S U(2)$ as a maximal subgroup $[20,21]$. Embed one of the $U(1)$ 's into $S U(2)$. This breaks $E_{8} \rightarrow E_{7}, E_{7}$ contains $E_{6} \times U(1)$ as a maximal subgroup. Identifying this $U(1)$ factor with the remaining $U(1)$ in $U(1) \times U(1)$ breaks $E_{7} \rightarrow E_{6}$. Under this breaking scheme the 248 of $E_{8}$ decomposes as

$$
\begin{aligned}
E_{8} \rightarrow \quad & E_{7} \times S U(2) \\
(248) \quad & (133,1)+(1,3)+(56,1)+(\overline{56}, 1) \\
\rightarrow E_{7} & \times U(1) \\
& (133)_{0}+(1)_{0}+(1)_{-2}+(1)_{2}+(56)_{1}+(\overline{56})_{-1} \\
\rightarrow E_{6} & \times U(1) \times U(1) \\
& (78)_{0,0}+(27)_{0,-2}+(\overline{27})_{0,2} \\
& +(1)_{0,0}+(1)_{-2,0}+(1)_{2,0} \\
& +(1)_{1,3}+(1)_{1,-3}+(27)_{1,1}+(\overline{27})_{1,-1} \\
& +(1)_{-1,3}+(1)_{-1,-3}+(\overline{27})_{-1,-1}+(27)_{-1,1}(70)
\end{aligned}
$$

The index theorem for fermions of charge $(1,1)$ yields

$n_{+}-n_{-}=\frac{1}{6} \int c_{1}^{3}-\frac{1}{24} \int c_{1} p_{1}$

since $c_{3}=0, c_{2}=0$ for $U(1)$ fibres. 
Let $x$ be the Kahler two form (volume form) on $S^{2}\left(x^{2}=0\right)$ and $y$ be the Kahler two form on $C P^{2}\left(y^{2}\right.$ $=$ volume form, $\left.y^{3}=0\right)$. Then $p_{1}=p_{1}\left(C P^{2}\right)=3 y^{2}$ [22] and $c_{1}=m x+n y$ where $m=$ monopole number of the $U$ (1) field on $S^{2}$ and $n=$ monopole number of the other $U(1)$ field on $C P^{2}$; then (71) reduces to

$\left(c_{1}^{3}=3 m n^{2} x y^{2}, \int x y^{2}=1\right)$

$n_{+}-n_{-}=\frac{1}{2} m n^{2}-\frac{1}{8} m$.

For fermions of charge $(p, q)$, this is modified to

$n_{+}-n_{-}=\frac{1}{2}(p m)(q n)^{2}-\frac{1}{8} p m$

when the $U(1)$ which breaks $E_{8} \times E_{7}$ lives on $S^{2}$ and the $U(1)$ which breaks $E_{7} \rightarrow E_{6}$ lives on $C P^{2}$. Alternatively

$n_{+}-n_{-}=\frac{1}{2}(q m)(p n)^{2}-\frac{1}{8} q m$

when the $U(1)$ 's change roles.

For a fermion of charge $(1,1)$ on $\frac{S U(3)}{U(1) \times U(1)}$, (72) shows that $m$ must be a multiple of 8 . This reflects the fact that $\frac{S U(3)}{U(1) \times U(1)}$ does not admit a spinor structure coupled to a $U(1) \times U(1)$ field unless $m$ is a multiple of 8 .

As an example we take the simplest non-trivial case $n=1, m=8$. From the decomposition (70) there are three different 27 's to consider,

$(p, q)=(0,-2),(1,1)$ and $(-1,1)$.

When the $U(1)$ field on $S^{2}$ is used to break $E_{8} \rightarrow E_{7}$, (73) gives

$n_{+}-n_{-}=p\left(4 q^{2}-1\right)$.

Hence the number of massless $(27)_{1,1}$ 's exceeds the $(\overline{27})_{-1,-1}$ 's by 3 , the number of massless $(\overline{27})_{1,-1}$ 's exceeds the number of $(27)_{-1,1}$ 's by 3 , and there is no imbalance between the $(27)_{0,-2}$ 's and the $(27)_{0,2}$ 's. If the difference in $U(1)$ charges shows up as a physical difference in four dimensions, it is possible that the $27_{1,-1}$ 's behave very differently from the $27_{1,1}$ 's which might give a chirally asymmetric theory in four dimensions.

When the $U(1)$ field on $C P^{2}$ is used for the first step $E_{8} \rightarrow E_{7}$, (74) gives

$n_{+}-n_{-}=q\left(4 p^{2}-1\right)$.

Hence the number of massless $(27)_{0,-2}$ 's exceeds the $(\overline{27})_{0,2}$ 's by 2 , the number of massless $(27)_{1,1}$ 's exceeds the (27) $)_{-1,-1}$ 's by 3 and the number of massless $(27)_{-1,1}$ 's exceeds the $(27)_{1,-1}$ 's by 3 . Thus we have a total imbalance of 8 massless (27)'s in four dimensions, though again the different $U(1) \times U(1)$ charges may give different physics for each in four dimensions.

All this looks very interesting for phonomenology, but unfortunately our ansatz for solving the dynamics has used $m=n$ on $\frac{S U(3)}{U(1) \times U(1)}$ and $m=8=n$ leads to an unacceptably large number of chiral fermion families. However, should it prove possible to relax this, the above scheme is an interesting alternative to previous proposals.

It is possible to contrive such a situation by having a $U(1) \times U(1) \times U(1)$ field where $U(1) \times U(1)$ is embedded within one $E_{8}$ as $m=8, n=1$ and the other is embedded within the other $E_{8}$ as $n=7$; then this will appear within the Einstein scalar equations as a $m=n=8$ case. The resultant gauge theory would be $E_{6} \times E_{7}$ and it would be possible (as described before) to have a realistic number of chiral 27's of $E_{6}$. We have discussed this symmetry breaking scheme in detail, because it may lead to interesting physics and it has not been examined in the literature. Other schemes have been explored in [5] and [19]. In particular the authors of [19] discuss a scheme with $E_{8}$ $\rightarrow S O(10)$ and 3 massless 16 's of $S O(10)$ in four dimensions.

\section{Discussion}

We have exhibited solutions of the modified Chapline-Manton Lagrangian with $F^{\bar{a}}=0$ in which four dimensional space-time is de Sitter, anti-de Sitter or unbounded Einstein static cosmology and the internal manifold is $\frac{G_{2}}{S U(3)}$ or $\frac{S U(3)}{U(1) \times U(1)}$, and solutions in which four dimensional space-time is Minkowski, anti-de Sitter, de Sitter or unbounded Einstein static cosmology with $F^{\bar{a}} \neq 0$ and defined on $\frac{G_{2}}{S U(3)}$, $\frac{S U(3)}{U(1) \times U(1)}$ or $\frac{S p(4)}{S U(2) \times U(1)}$ The problems with $\frac{S p(4)}{S U(2) \times U(1)}$ when $F^{\bar{a}}=0$ were also encountered in [7]. When $F^{a} \neq 0$, the Einstein static cosmology has a one parameter family of solutions which joins smoothly onto four dimensional Minkowski space and one may envisage an evolutionary process in which the parameter $\beta$ changes with time and the four dimensional universe evolves from a very highly curved $\mathbb{R} \times(\mathrm{Hyp})^{3}$ to four dimensional Minkowski space, with only very slight variations in the internal curvature. A quantitative analysis of the details of such a process is however beyond the scope of this work. 
For a Minkowski space solution, we need to have the torsion on the internal manifold. We find the value $\beta=-2.13$ is necessary to solve the equations of motion. This differs from both the Ricci flattening value $\beta=1 \pm \sqrt{5}[6]$ and the value necessary for the cancellation of conformal anomalies in the $\sigma$-model, $\beta=1-\sqrt{15}[7]$.

The presence of the $U(1)$ factors in $V$ results in many possible chiral fermion spectra in four dimensions for $\frac{S U(3)}{U(1) \times U(1)}$ and $\frac{S p(4)}{S U(2) \times U(1)}$. Embedding $V$ into one of the $E_{8}$ 's of the $E_{8} \times E_{8}$ superstring theory can result in an effective $E_{6}$ theory in four dimensions. For $\frac{G_{2}}{S U(3)}$ we get one family of a chiral 27 of fermions in the usual way [2], [23]. For $\frac{S U(3)}{U(1) \times U(1)}$ we can get three 27 's and $\overline{27}$ 's each with different $U(1)$ quantum numbers or eight 27's depending on how we perform the embedding. Perhaps the most promising case for phenomenology is $\frac{S p(4)}{S U(2) \times U(1)}$ where we can get any even integer for the number of 27's in four dimensions, depending on the winding number of the $U(1)$ into the maximal torus of $S p(4)$. Apart from a qualitative remark about $\frac{G_{2}}{S U(3)}$ we have not discussed the possible role of the isometry group $W$.

We have not discussed the residual supersymmetry of these background configurations (for $\frac{G_{2}}{S U(3)}$ this is tackled in Ref. [23]) for the following reason: the Lagrangian $\mathscr{L}+\Delta \mathscr{L}$ is incomplete as a low energy expansion of the superstring. To be consistent, we should include all terms up to fourth order in derivatives e.g. $H^{4}, F^{4}, H^{2} F^{2}, H^{2} R^{a b}$ etc., coming from the superstring. We do not know what these terms are, nor what their effect on the supersymmetry transformations would be. The addition of $\triangle \mathscr{L}$ to $\mathscr{L}$ spoils the supersymmetry transformations of ChaplineManton [9] and we do not yet know how to modify them to a consistent order in $\alpha^{\prime} E^{2}$ to restore supersymmetry.

Acknowledgements. We wish to thank R.G. Moorhouse for helpful discussions. One of us (A.B.H.) would like to thank the Department of Physics and Astronomy at Glasgow University for their hospitality.

\section{Appendix}

$\frac{G_{2}}{S U(3)}$

The structure constants of $G_{2}$ are:

$$
\begin{aligned}
& C_{23}{ }^{4}=\frac{1}{2 \sqrt{3}}, C_{13}{ }^{5}=\frac{1}{2 \sqrt{3}}, \\
& C_{45}{ }^{6}=-\frac{1}{2 \sqrt{3}}, C_{12}{ }^{6}=-\frac{1}{2 \sqrt{3}} \\
& C_{15}{ }^{9}=-\frac{1}{4}, \quad C_{16}{ }^{7}=-\frac{1}{4}, \\
& C_{23}{ }^{11}=-\frac{1}{4}, \quad C_{26}{ }^{8}=-\frac{1}{4} \\
& C_{35}{ }^{8}=-\frac{1}{4}, \quad C_{45}{ }^{12}=-\frac{1}{4}, \\
& C_{13}{ }^{10}=\frac{1}{4}, \quad C_{12}{ }^{12}=\frac{1}{4} \\
& C_{24}{ }^{9}=\frac{1}{4}, \quad C_{46}{ }^{10}=-\frac{1}{4} \text {, } \\
& C_{34}{ }^{7}=\frac{1}{4}, \quad C_{56}{ }^{11}=-\frac{1}{4} \\
& C_{25}{ }^{13}=-\frac{1}{4}, \quad C_{36}{ }^{13}=\frac{1}{4}, \\
& C_{9,12}{ }^{13}=\frac{1}{4}, \quad C_{7,10}{ }^{13}=-\frac{1}{4} \\
& C_{78}{ }^{12}=\frac{1}{4}, \quad C_{79}{ }^{11}=\frac{1}{4} \text {, } \\
& C_{89}{ }^{10}=-\frac{1}{4}, \quad C_{10,11}{ }^{12}=-\frac{1}{4} \\
& C_{25}{ }^{14}=\frac{1}{4 \sqrt{3}}, \quad C_{36}{ }^{14}=\frac{1}{4 \sqrt{3}}, \\
& C_{7,10}{ }^{14}=\frac{\sqrt{3}}{4}, \quad C_{9,12}{ }^{14}=\frac{\sqrt{3}}{4}^{3} \\
& C_{8,11}{ }^{13}=\frac{1}{2}, \quad C_{14}{ }^{14}=\frac{1}{2 \sqrt{3}}
\end{aligned}
$$

$7, \ldots, 14$ label the generators of $S U(3)$.

The normalisation is chosen so that $C_{\hat{a} \hat{b}}^{\hat{b}} C_{\hat{d} \hat{c}}^{\hat{b}}=$ $-\delta_{\hat{a} \hat{d}}$ and indices can be raised and lowered with no change.

With this normalisation the $S U(3)$ structure constants satisfy $C_{\bar{a} \bar{b}} \bar{c} C_{\bar{d} \bar{c}}^{\bar{b}}=-\frac{3}{4} \delta_{\bar{a} \bar{d}}$.

Hence, in the adjoint representation

$$
\operatorname{Tr}_{G_{2}}\left(Q_{\hat{a}} Q_{\hat{b}}\right)_{a d j}=-\delta_{\hat{a} \hat{d}}, \quad \operatorname{Tr}_{S U(3)}\left(Q_{a} Q_{\bar{b}}\right)_{a d j}=-\frac{3}{4} \delta_{\bar{a} \bar{d}} .
$$

The ratio, $\frac{3}{4}$, of these traces is independent of the normalisation used for the structure constants and is, by definition the ratio of the second index of the representations, $I_{2}\left[\operatorname{adj}\left(G_{2}\right)\right]=8, \quad I_{2}[\operatorname{adj}(S U(3))]=6 \quad[3$, 21]. In the fundamental representation $I_{2}[$ fund $(S U(3))]=1$, hence

$\operatorname{tr}_{S U(3)}\left(Q_{\tilde{a}} Q_{\bar{b}}\right)_{\text {fund }}=-\frac{1}{8} \delta_{\bar{a} \bar{b}}$.

When embedding $S U(3)$ into $E_{8}$, we need to use the fact that $I_{2}\left[\right.$ fund $\left.\left(E_{8}\right)\right]=I_{2}\left[\operatorname{adj}\left(E_{8}\right)\right]=60$ 
$\therefore \frac{1}{30} \operatorname{Tr}_{E_{8}}\left(Q_{\bar{a}} Q_{\bar{b}}\right)_{\text {fund }}$

$=\frac{1}{30} \frac{I_{2}\left[\text { fund }\left(E_{8}\right)\right]}{I_{2}[\text { fund }(S U(3)]} \operatorname{tr}_{S U(3)}\left(Q_{\bar{a}} Q_{\bar{b}}\right)_{\mathrm{fund}}=-\frac{1}{4} \delta_{\bar{a} \bar{b}}$.

So $N^{2}=\frac{1}{4}$.

In the text, $K$ and $L$ are defined by

$$
\begin{aligned}
& K \delta^{a b}=C_{c d}{ }^{a} C^{c d b}, \quad L \delta^{\bar{a} \bar{b}}=C_{c d}{ }^{\bar{a}} C^{c d \bar{b}} \\
\Rightarrow & K=\frac{1}{3}, \quad L=\frac{1}{4}
\end{aligned}
$$

$v=\operatorname{dim} V=8$ for $S U(3)$, hence $A$ as defined in (38) is given by

$A=11-12 N^{2} ; \quad A=11$ when $\quad F^{\bar{a}}=0, \quad A=8$

when $\quad F^{a} \neq 0$.

Note that $\beta=1$ gives $R^{a b}=\frac{\alpha^{2}}{12} e^{a} \wedge e^{b}$, the standard torsion free Riemannian curvature two forms on $S^{6}$ with $S O(6)$ holonomy.

\section{$\frac{S U(3)}{U(1) \times U(1)}$}

The structure constants of $S U(3)$ are

$$
\begin{array}{ll}
C_{12}{ }^{6}=-\frac{1}{2 \sqrt{3}}, & C_{13}{ }^{5}=-\frac{1}{2 \sqrt{3}}, \\
C_{23}{ }^{4}=-\frac{1}{2 \sqrt{3}}, & C_{45}{ }^{6}=-\frac{1}{2 \sqrt{3}} \\
C_{25}{ }^{7}=-\frac{1}{2 \sqrt{3}}, & C_{36}{ }^{7}=\frac{1}{2 \sqrt{3}}, \\
C_{14}{ }^{7}=-\sqrt{3}, & C_{25}{ }^{8}=-\frac{1}{2}, \\
C_{36}{ }^{8}=-\frac{1}{2} &
\end{array}
$$

The $U(1)$ generators are numbers 7 and 8 .

The normalisation is such that $C_{\hat{a} \hat{b}}^{\hat{c}} C_{\hat{d} \hat{c}}^{\hat{b}}=\delta_{\hat{a} \hat{d}}$.

The $U(1)$ generators, when embedded into $E_{8}$, are normalised so that

$$
\begin{aligned}
& \frac{1}{30} \operatorname{Tr}_{E_{8}}\left(Q_{7} Q_{7}\right)_{\text {fund }}=\frac{1}{30} \operatorname{Tr}_{E_{8}}\left(Q_{8} Q_{8}\right)_{\mathrm{fund}}=-1 . \\
\Rightarrow & N^{2}=m^{2}
\end{aligned}
$$

where $m$ is the monopole number for the $U(1)$ fields over $S^{2}$ and $C P^{2}$ respectively (see discussion for (72) and (74), with $m=n$ ). With these structure constants

$$
\begin{aligned}
K & =\frac{1}{3}, \quad L=1, \quad v=2 \\
\Rightarrow A & =20 \quad \text { when } \quad F^{\bar{a}}=0, \quad A=8 \quad \text { when } \quad F^{\bar{a}} \neq 0, \\
m & =n=1
\end{aligned}
$$

$\frac{S p(4)}{S U(2) \times U(1)}$

The structure constants of $S p(4)$ are

$$
\begin{aligned}
C_{126} & =-C_{135}=C_{234}=-C_{456}=-C_{238}=-C_{259} \\
& =\frac{1}{\sqrt{12}} \\
C_{357} & =C_{568}=-C_{25,10}=-C_{369}=-C_{36,10}=C_{267} \\
& =-\frac{1}{\sqrt{12}} \\
C_{14,10} & =C_{789}=-\frac{1}{\sqrt{3}}
\end{aligned}
$$

$7,8,9$ label the generators of $S U(2)$ and 10 the generator of $U(1)$. The normalisation is chosen so that

$\operatorname{Tr}_{S p(4)}\left(Q_{\hat{a}} Q_{\hat{b}}\right)_{\mathrm{adj}}=+C_{\hat{a} \hat{c}}^{\hat{d}} C_{\hat{b} \hat{d}}^{\hat{c}}=-\delta_{\hat{a} \hat{b}}$.

For the $S U(2)$ subgroup

$\operatorname{Tr}_{S U(2)}\left(Q_{\bar{a}} Q_{\bar{b}}\right)$

$=\frac{I_{2}[\operatorname{adj}(S U(2))]}{I_{2}[\operatorname{adj}(S p(4))]} \operatorname{tr}_{S p(4)}\left(Q_{\bar{a}} Q_{\bar{b}}\right)_{\mathrm{adj}}=-\frac{2}{3} \delta_{\bar{a} \bar{b}}$.

Since $I_{2}[\operatorname{adj}(S U(2))]=4$ and $I_{2}[\operatorname{adj}(S p(4))]=6$, see [3].

In the fundamental representation

$I_{2}[$ fund $(S U(2))]=1$ so we find

$\operatorname{tr}_{S U(2)}\left(Q_{\bar{a}} Q_{\bar{b}}\right)_{\mathrm{fund}}=-\frac{1}{6} \cdot \delta_{\bar{a} \bar{b}}$.

When embedding $S U(2)$ into $E_{8}$ we find

$\frac{1}{30} \operatorname{Tr}_{E_{\mathbf{8}}}\left(Q_{\bar{a}} Q_{\bar{b}}\right)_{\text {fund }}$

$=\frac{1}{30} \frac{I_{2}\left[\text { fund }\left(E_{8}\right)\right]}{I_{2}[\text { fund }(S U(2)]} \operatorname{tr}_{S U(2)}\left(Q_{\bar{a}} Q_{\bar{b}}\right)_{\mathrm{fund}}=-\frac{1}{3} \delta_{\bar{a} \bar{b}}$.

And so $N^{2}=\frac{1}{3}$.

These structure constants give $K=\frac{1}{3}$ but $L$ is a little subtle because,

$C_{a b}{ }^{\bar{c}} C^{a b \bar{d}}=\frac{1}{3} \delta^{\bar{c} \bar{d}} \quad$ for $\bar{c}, \bar{d}=7,8,9$ and $\quad C_{a b}{ }^{10} \mathrm{C}^{\mathrm{ab} 10}=1$.

This causes various difficulties with our ansatz since we explicitly used the fact that $C_{a b \bar{c}} C^{a b \vec{a}}=L \delta_{\bar{c}}^{\vec{d}}$ in several places. There are two areas of difficulty:

1) In the $d H=\frac{\operatorname{Tr}}{30} F^{2}-\operatorname{tr} R^{2}$ equation we find $\operatorname{tr} R^{2}$ is not proportional to $d H$.

2) In the Einstein equations $R_{a b} \wedge R_{c d} \wedge i^{e}\left(* e^{a b c d}\right)$ is no longer a (const) $\times * e^{e}$ for $e$ an internal space index. This causes difficulties with the Einstein equations. 
We find, however, that if we have a $S U(2) \times U(1)$ Yang-Mills field and the generators are normalised so that $N^{2}=\frac{1}{3}$ for the $S U(2)$, and $N^{2}=1$ for the $U(1)$ (these are the expected values), then the $F^{2}$ terms also suffer from problems 1) and 2); however, when we consider the equation $d H=\frac{1}{30} \operatorname{Tr} F^{2}-\operatorname{tr} R^{2}$, we find in the RHS that we have a cancellation of the parts not proportional to $d H$. We also find that the energymomentum for the $R$ and $F$ fields considered together poses no difficulties in the Einstein equations. So, for the $\frac{S p(4)}{S U(2) \times U(1)}$ case, we really need the $F$ and $R$ fields together to make our ansatz work. We find we should take $A=8$ in (38). Notice that we might have expected solutions with multiple monopole number for the $U(1)$ but we cannot allow this without disturbing the Bianchi identity.

\section{References}

1. J.H. Schwarz: Introduction to superstrings, in: Superstrings and supergravity. Proceedings of the 28th Scottish Universities Summer School in Physics (1985). A.T. Davies, D.G. Sutherlands (eds.)

2. P. Candelas, G.T. Horowitz, A. Strominger, E. Witten: Nucl. Phys. B 258 (1985) 46

3. K. Piich, A.N. Schellekens: Nucl. Phys. B259 (1985) 637

4. G. Chapline, B. Grossman: Phys. Lett. 135 B (1984) 109

5. G. Chapline, B. Grossman: Phys. Lett. 143 B (1984) 161

6. D. Lüst: Nucl. Phys. B276 (1986) 220

7. L. Castellani, D. Lüst: Superstring compactification on homogeneous coset spaces with torsion, Caltec., preprint CALT-86-1353

8. P.G.O. Freund, M.A. Rubin: Phys. Lett. 97 B (1980) 233
9. G.F. Chapline, N.S. Manton: Phys. Lett. $120 \mathrm{~B}$ (1983) 105. A Chamseddine: Phys. Rev. D24 (1981) 3065; E. Bergshoeff, M. de Roo, B. de Wit, P. Van Nieuwenhuizen: Nucl. Phys. B195 (1982) 97

10. B. Zweibach: Phys. Lett. 156B (1985) 315

11. D.Z. Freedman, G. Gibbons, P.C. West: Phys. Lett. 124 B (1983) 491

12. M.G. Green, J.H. Schwarz: Phys. Lett. 149 B (1984) 117

13. A. Salam, J. Strathdee: Ann. Phys. (NY) 141 (1982) 316

14. S. Kobayashi, K. Nomizu: Foundations of differential geometry (Vol. II) New York: Wiley 1969

15. E. Witten: Phys. Lett. 149 B (1984) 351

16. I.R. Porteous: Topological geometry. 2nd edit. Cambridge: Cambridge University Press 1981

17. K.A. Olive, D.N. Schramm, G. Steigmann, M.S. Turner: Phys. Lett. 176B (1986) 33

18. R. Bott, L.W. Tu: Differential forms in algebraic topology. Springer, Berlin, Heidelberg, New York : 1982

19. K. Pilch, A.N. Schellekens: Phys. Lett. 164 B (1985) 31

20. R. Slansky: Phys. Rep. 79 (1981) 1

21. W.G. McKay, J. Patera: Tables of dimensions, indices and branching rules for representations of simple lie algebras. New York, Basel: Dekker 1981

22. T. Eguchi, P.B. Gilkey, A.J. Hanson: Phys. Rep. 66 (1980) 213

23. G. Fogleman, K.S. Viswanathan, B. Wong: Super Field Theories. H.C. Lee, V. Elias, G. Kunstatter, R.B. Mann, K.S Viswanathan (eds.) NATO ASI Series B, Vol 160, New York: Plenum 1987

24. S. Randjbar-Daemi, A. Salam, J. Strathdee: Phys. Lett. 124B (1983) 349

25. K. Maeda, P.Y.T. Pang: Phys. Lett 180 B (1986) 29

26. K. Maeda, M.D. Pollock: Phys. Lett. 173 B (1986) 251

27. G.F. Chapline: Class. Quant. Grav. 3 (1986) L 35

28. I. Bars: Phys. Rev. D 33 (1986) 383

29. C.M. Hull: Phys. Lett 178 B (1986) 357

30. R. Rohm, E. Witten: Ann. Phys. 170 (1986) 454

31. T.R. Govindarajan, A.S. Joshipura, S.D. Rindani, U. Sarkar: Phys. Lett. 57 (1986) 2487, Int. J. Mod. Phy. A2 (1987) 797 